\title{
A geometric construction of travelling wave solutions to a Keller-Segel model
}

\author{
K. Harley ${ }^{1} \quad$ P. van Heijster ${ }^{2} \quad$ G. J. Pettet ${ }^{3}$
}

(Received 17 December 2013; revised 8 August 2014)

\begin{abstract}
We study a version of the Keller-Segel model for bacterial chemotaxis for which explicit travelling wave solutions are known in the zero attractant-diffusion limit. Travelling wave solutions are constructed in the small diffusion case using geometric singular perturbation theory, which converge to the explicit solutions in the singular limit.
\end{abstract}

\section{Contents}

\section{Introduction}

1.1 An explicit solution for $\mathrm{D}_{\mathfrak{u}}=0$

1.2 Taking the limit as $\mathrm{D}_{w} \rightarrow 0$
$\mathrm{C} 400$

$\mathrm{C} 402$

C402

http://journal.austms.org.au/ojs/index.php/ANZIAMJ/article/view/7801 gives this article, (c) Austral. Mathematical Soc. 2014. Published August 30, 2014, as part of the Proceedings of the 11th Biennial Engineering Mathematics and Applications Conference. ISSN 1446-8735. (Print two pages per sheet of paper.) Copies of this article must not be made otherwise available on the internet; instead link directly to this URL for this article. 
2 Travelling wave solutions for $0<\mathrm{D}_{u, w} \ll 1$

C404

2.1 Layer problem . . . . . . . . . . . . . . . . . . C406

2.2 Reduced problem . . . . . . . . . . . . . . . . . C409

2.3 Singular heteroclinic orbits . . . . . . . . . . . C410

2.4 Heteroclinic orbits for $0<\varepsilon \ll 1 \ldots \ldots$. . . . . . C410

3 Conclusion

C412

References

C413

\section{Introduction}

The Keller-Segel model $[11,12]$ is a very popular model for cell migration in response to a chemical gradient [7, 13, e.g., and references therein]. Because it has explicit travelling wave solutions in the limit where diffusion $\mathrm{D}_{\mathfrak{u}} \rightarrow 0$ [3], we are interested in the following particular version of the Keller-Segel model:

$$
\begin{aligned}
\frac{\partial u}{\partial t} & =D_{u} \frac{\partial^{2} u}{\partial x^{2}}-K w, \\
\frac{\partial w}{\partial t} & =D_{w} \frac{\partial^{2} w}{\partial x^{2}}-\frac{\partial}{\partial x}\left(\frac{Q w}{u} \frac{\partial u}{\partial x}\right),
\end{aligned}
$$

with $u>0, w \geqslant 0, x \in \mathbb{R}, t>0, K, Q>0, D_{u, w} \geqslant 0$. Here $u(x, t)$ is the concentration of the chemical or chemoattractant and $w(x, t)$ is the density of the migrating species. The constant $\mathrm{K}$ is the rate of uptake of the chemical by the migrating species, the constant $\mathrm{Q}$ is the chemotactic coefficient and the constants $\mathrm{D}_{\mathfrak{u}, w}$ are the diffusivities of each species. In particular, we are interested in finding travelling wave solutions to (1) in the case where both the diffusivities are small but of the same order: $0 \leqslant D_{u, w} \ll 1$.

With $\mathrm{D}_{\mathfrak{u}, w}$ small, (1) is a singularly perturbed system; due to the advection (chemotaxis) term (the last term in the $w$ equation in (1)) we are unable to scale out the small parameters. This makes (1) amenable for analysis via 
geometric singular perturbation theory (GSPT) $[8,10]$. Using GSPT, we show that (1) supports travelling wave solutions, which, in the limit $\mathrm{D}_{\mathfrak{u}, w} \rightarrow 0$, agree with the explicit solutions given by Feltham and Chaplain [3].

The background states of $(1)$ are $(\mathfrak{u}, \boldsymbol{w})=\left(\mathbf{u}^{*}, 0\right)$, with $\mathbf{u}^{*} \geqslant 0$ for physically relevant solutions. We are interested in travelling wave solutions that travel with a constant speed $\mathrm{c}$. Therefore, we introduce a comoving frame $z=x-c t$ and (1) becomes

$$
\begin{aligned}
-c \frac{d u}{d z} & =D_{u} \frac{d^{2} u}{d z^{2}}-K w \\
-c \frac{d w}{d z} & =D_{w} \frac{d^{2} w}{d z^{2}}-\frac{d}{d z}\left(\frac{Q w}{u} \frac{d u}{d z}\right) .
\end{aligned}
$$

In particular, we study travelling wave solutions that satisfy the asymptotic conditions

$$
\lim _{z \rightarrow-\infty} \mathfrak{u}(z)=\mathfrak{u}_{l}, \quad \lim _{z \rightarrow \infty} \mathfrak{u}(z)=\mathfrak{u}_{\mathrm{r}}>\mathfrak{u}_{l}, \quad \lim _{z \rightarrow \pm \infty} w(z)=0 .
$$

Assuming $\mathfrak{u}_{\mathrm{r}}>\mathfrak{u}_{\mathfrak{l}}$ implies $\boldsymbol{c}>0$; integrating the first equation of (2) with respect to $z$ from $-\infty$ to $\infty$ and applying the asymptotic conditions for $u$ along with $w \geqslant 0$ gives $c\left(u_{r}-u_{l}\right)>0$.

Integrating the second equation of (2) gives

$$
\sigma_{0}=D_{w} \frac{d w}{d z}+c w-\frac{Q w}{u} \frac{d u}{d z}
$$

with $\sigma_{0}$ a constant of integration. When $z \rightarrow \infty,\left(u(z), w(z), u^{\prime}(z), w^{\prime}(z)\right) \rightarrow$ $\left(u_{r}, 0,0,0\right)$ with $u_{r}>0$ by definition. This implies $\sigma_{0}=0$ [3]. Thus, the system considered henceforth is

$$
\begin{aligned}
& \mathrm{D}_{\mathrm{u}} \frac{\mathrm{d}^{2} \mathrm{u}}{\mathrm{d} z^{2}}=\mathrm{K} w-\mathrm{c} \frac{\mathrm{du}}{\mathrm{d} z}, \\
& \mathrm{D}_{w} \frac{\mathrm{d} w}{\mathrm{~d} z}=\frac{\mathrm{w} w}{\mathrm{u}} \frac{\mathrm{du}}{\mathrm{d} z}-\mathrm{cw},
\end{aligned}
$$

along with (3). 


\subsection{An explicit solution for $D_{\mathfrak{u}}=0$}

As alluded to above, for $\mathrm{D}_{\mathfrak{u}}=0$ and $\mathrm{D}_{w}<\mathrm{Q}$, (2) (and hence (4)) has explicit solutions [3, for derivation]:

$$
\begin{aligned}
\mathrm{U}(z) & :=\left[\mathrm{u}_{\mathrm{r}}^{-1 / \gamma}+\sigma \mathrm{e}^{-\mathrm{c}\left(z+z^{*}\right) / \mathrm{D}_{w}}\right]^{-\gamma} \\
\mathrm{W}(z) & :=\mathrm{e}^{-\mathrm{c}\left(z+z^{*}\right) / \mathrm{D}_{w}}\left[\mathrm{u}_{\mathrm{r}}^{-1 / \gamma}+\sigma \mathrm{e}^{-\mathrm{c}\left(z+z^{*}\right) / \mathrm{D}_{w}}\right]^{-\gamma \mathrm{Q} / \mathrm{D}_{w}},
\end{aligned}
$$

with

$$
\gamma=\frac{\mathrm{D}_{w}}{\mathrm{Q}-\mathrm{D}_{w}}>0, \quad \sigma=\frac{\mathrm{K}\left(\mathrm{Q}-\mathrm{D}_{w}\right)}{\mathrm{c}^{2}}>0
$$

and $z^{*}$ an integration constant representing the translational invariance of the travelling wave solutions.

For fixed $0<\mathrm{D}_{w}<\mathrm{Q}, \lim _{z \rightarrow-\infty} \mathrm{U}(z)=0$, with $\mathrm{U}(z)$ defined in (5). Thus, in order to compare solutions of (4) with the explicit solution $(U, W)(z)$ (defined in (5)), we take $\mathfrak{u}_{\mathfrak{l}}=0$. Furthermore, since (4) is invariant with respect to the multiplicative scaling

$$
(\mathfrak{u}, w) \rightarrow(k u, k w), \quad k \in \mathbb{R},
$$

we assume, without loss of generality, $\mathfrak{u}_{\mathrm{r}}=1$.

Remark 1. Observe that

$$
\lim _{z \rightarrow-\infty} \frac{W(z)}{U(z)}=\frac{c^{2}}{K\left(Q-D_{w}\right)},
$$

so the $w / u$ term in (4) is bounded for the explicit solution as $z \rightarrow-\infty$.

\subsection{Taking the limit as $\mathrm{D}_{w} \rightarrow 0$}

Since we are interested in the case where both diffusivities are small, consider the limit of $(5)$ as $\mathrm{D}_{w} \rightarrow 0$. Choosing $z^{*}=\left(\mathrm{Q}-\mathrm{D}_{w}\right) \ln \left(\mathrm{u}_{\mathrm{r}}\right) / \mathrm{c}$ with $\mathrm{u}_{\mathrm{r}}=1$ 


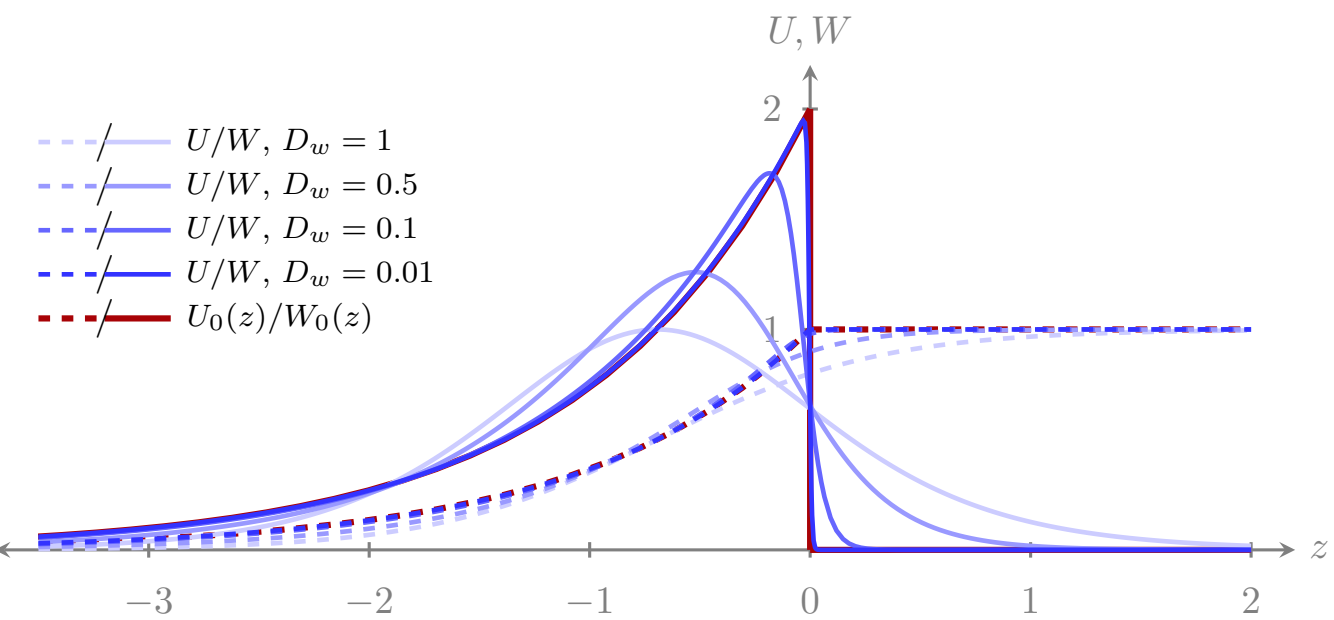

Figure 1: Plots of $(U, W)(z)$ defined in (5), with decreasing $D_{w}$, as well as $\left(U_{0}, W_{0}\right)(z)$, defined in $(7)$. The dashed curves correspond to $U(z)$ and the solid curves to $W(z)$, with the value of $D_{w}$ indicated by the colour. We choose $z^{*}=\left(\mathrm{Q}-\mathrm{D}_{w}\right) \ln \left(\mathrm{u}_{\mathrm{r}}\right) / \mathrm{c}$ such that the shock in the $\mathrm{W}_{0}$-profile occurs at $z=0$. The remaining system parameters are given by Feltham and Chaplain [3]: $\mathrm{Q}=2, \mathrm{~K}=1, \mathrm{c}=2$ and $\mathrm{u}_{\mathrm{r}}=1$.

(that is, $z^{*}=0$ ) and evaluating the limit gives

$$
\lim _{\mathrm{D}_{w} \rightarrow 0} \mathrm{U}(z)=\left\{\begin{array}{ll}
\mathrm{e}^{\mathrm{c} z / \mathrm{Q}}, & z \leqslant 0, \\
1, & z>0,
\end{array} \quad \text { and } \quad \lim _{\mathrm{D}_{w} \rightarrow 0} \mathrm{~W}(z)= \begin{cases}\frac{\mathrm{c}^{2}}{\mathrm{KQ}} e^{\mathrm{c} z / \mathrm{Q}}, & z \leqslant 0 \\
0, & z>0\end{cases}\right.
$$

For convenience, we label this limit $\left(\mathrm{U}_{0}, \mathrm{~W}_{0}\right)$ and note that it is not a solution to (4) in the classical sense since the $W_{0}$-profile is discontinuous at $z=0$. This location of the discontinuity explains our choice of $z^{*}$ : a different $z^{*}$ would result in a similar, but translated, profile with the shock located at another $z$ value.

Figure 1 shows $(U, W)(5)$ for the above stated $z^{*}$ and decreasing $D_{w}$, as well 
as the shock solution $\left(\mathrm{U}_{0}, \mathrm{~W}_{0}\right)(7)$. Clearly, $(\mathrm{U}, \mathrm{W}) \rightarrow\left(\mathrm{U}_{0}, \mathrm{~W}_{0}\right)$ as $\mathrm{D}_{w} \rightarrow 0$. We provide evidence that (4) also possesses heteroclinic solutions connecting $(0,0)$ to $(1,0)$, with small diffusion coefficients $D_{u, w}$ (that is, $D_{\mathfrak{u}} \neq 0$ ). Therefore, we show that (1) supports travelling wave solutions connecting $(0,0)$ to $(1,0)$ and hence, by the scaling $(6)$, travelling wave solutions connecting $(0,0)$ to $\left(u_{r}, 0\right)$ with $u_{r}>0$.

\section{Travelling wave solutions for $0<\mathrm{D}_{\mathfrak{u}, w} \ll 1$}

Theorem 2. Let $\mathrm{D}_{\mathrm{u}}=\mu \varepsilon$ and $\mathrm{D}_{w}=\varepsilon$, with $0<\varepsilon \ll 1$ a sufficiently small parameter. Moreover, let $\mu, \mathrm{K}, \mathrm{Q}, \mathrm{c}$ be $\mathcal{O}(1)$ (with respect to $\varepsilon$ ) and positive constants. Then, travelling wave solutions to (1) connecting $(0,0)$ to $\left(\mathrm{u}_{r}, 0\right)$ with $\mathrm{u}_{\mathrm{r}}>0$, exist.

We use geometric singular perturbation theory (GSPT) to underpin the above theorem. GSPT is applied to problems exhibiting a clear separation of scales, for example, cell migration where diffusion is operating on a much slower scale than advection or reaction. The power of this method lies in the ability to separate the scales into independent, generically lower dimensional problems, which are more amenable to analysis.

We begin by introducing $v=\mathfrak{u}_{z}$ so (4) is rewritten as the three dimensional system of first order ordinary differential equations (ODEs),

$$
\begin{aligned}
\mathrm{u}_{z} & =v, \\
\mu \varepsilon v_{z} & =-c v+\mathrm{K} w, \\
\varepsilon \mathcal{W}_{z} & =-\mathrm{cw}+\frac{\mathrm{Q} v w}{\mathrm{u}} .
\end{aligned}
$$

This system exhibits two distinct spatial scales: the $\varepsilon$ premultiplying the $v$ and $w$ derivatives makes these fast variables, while $u$ is a slow variable, with $z$ the slow travelling wave coordinate. We refer to (8) as the slow system. 
Now we write (8) in terms of a fast travelling wave coordinate $\mathbf{y}=z / \varepsilon(\varepsilon \neq 0)$,

$$
\begin{aligned}
\mathrm{u}_{\mathrm{y}} & =\varepsilon v, \\
\mu v_{y} & =-c v+\mathrm{K} w, \\
w_{y} & =-c w+\frac{\mathrm{Q} v w}{u},
\end{aligned}
$$

which is referred to as the fast system.

In the singular limit $\varepsilon \rightarrow 0$, the slow system reduces to the one dimensional system with two algebraic constraints,

$$
\begin{aligned}
\mathrm{u}_{z} & =v, \\
0 & =-\mathrm{cv}+\mathrm{Kw}, \\
0 & =-\mathrm{cw}+\frac{\mathrm{Q} v w}{\mathrm{u}},
\end{aligned}
$$

which we call the reduced problem. Similarly, the fast system reduces to the two dimensional system with a parameter $\mathfrak{u}=\tilde{u} \in \mathbb{R}^{+}$,

$$
\begin{aligned}
\mu v_{y} & =-c v+K w \\
w_{y} & =-c w+\frac{Q v w}{\tilde{u}},
\end{aligned}
$$

which we refer to as the layer problem. In contrast to the slow and fast systems, the reduced and layer problems are not equivalent.

The strategy is as follows. Firstly, we analyse the two lower dimensional systems (10) and (11) independently. Secondly, we construct singular solutions that are concatenations of solutions of each of the subsystems. Finally, using GSPT, and in particular Fenichel theory [4, 5], we provide evidence that these singular solutions persist as solutions of the full three dimensional problem (8) or (9), and hence as travelling wave solutions of (1), for sufficiently small $0<\varepsilon \ll 1$. 


\subsection{Layer problem}

The layer problem (11) is a system of two first order ODEs with parameters $\mathrm{K}, \mathrm{Q}, \mathrm{c}, \mu, \tilde{\mathrm{u}}>0$. It has two steady states $(v, w)=\left(v^{\mathrm{n}}, w^{\mathrm{n}}\right)=(0,0)$ and $(v, w)=\left(v^{s}, w^{s}\right)=\left(c \tilde{u} / Q, c^{2} \tilde{u} /(Q K)\right)$. The steady states coincide in the limit $\tilde{u} \rightarrow 0$.

Lemma 3. For all $\mathrm{K}, \mathrm{Q}, \mathrm{c}, \mu, \tilde{\mathrm{u}}>0$, the layer problem (11) possesses a heteroclinic orbit connecting $\left(v^{\mathrm{s}}, w^{\mathrm{s}}\right)$ with $\left(v^{\mathrm{n}}, w^{\mathrm{n}}\right)$. We refer to this connection as a fast fibre.

Proof: The steady state $\left(v^{\mathfrak{n}}, w^{\mathfrak{n}}\right)$ is a stable node with eigenvalues and eigenvectors

$$
\begin{array}{ll}
\lambda_{1}^{\mathfrak{n}}=-\mathrm{c} / \mu, & \boldsymbol{\psi}_{1}^{\mathrm{n}}=(1,0), \\
\lambda_{2}^{\mathfrak{n}}=-\mathrm{c}, & \boldsymbol{\psi}_{2}^{\mathrm{n}}=(\mathrm{K}, \mathrm{c}(1-\mu)) .
\end{array}
$$

For $\mu=1$ the eigenvalues coincide and have algebraic multiplicity two. The generalised eigenvector in this situation is $\boldsymbol{\psi}_{2}^{g}=(0,1 / \mathrm{K})$. The other steady state $\left(v^{\mathfrak{s}}, \mathcal{w}^{\mathrm{s}}\right)$ is a saddle with eigenvalues and eigenvectors

$$
\begin{array}{ll}
\lambda_{1}^{s}=c \theta^{-} / \mu>0, & \psi_{1}^{s}=\left(K \theta^{-}, c \mu\right), \\
\lambda_{2}^{s}=-c \theta^{+} / \mu<0, & \psi_{2}^{s}=\left(-K \theta^{+}, c \mu\right),
\end{array}
$$

where $\theta^{ \pm}=\frac{1}{2} \sqrt{1+4 \mu} \pm \frac{1}{2}$. With the exception of the location of the steady state $\left(v^{\mathrm{s}}, w^{\mathrm{s}}\right)$, these linearisations are independent of $\tilde{u}$.

The $v$-nullcline of $(11)$ is $w=c v / K$ and the $w$-nullclines are $w=0$ and $v=c \tilde{u} / Q$. Since $\mu>\theta^{-}$for $\mu>0$ (which is a trivial exercise to show), the unstable trajectory leaving $\left(v^{s}, w^{s}\right)$ in the downward direction enters the region $\mathcal{R}:=\{(v, w) \mid 0<v<c \tilde{u} / Q, 0<w<c v / K\}$, the region enclosed by the three nullclines. The trivial $\mathcal{W}$-nullcline (the $v$-axis) is invariant with respect to the flow of (11). On the other boundaries of $\mathcal{R}$, the flow of (11) points inward. This yields that trajectories entering $\mathcal{R}$ cannot leave $\mathcal{R}$. Thus, as the stable trajectory entering $\left(\nu^{s}, w^{s}\right)$ is not in $\mathcal{R}$ (that is, $\theta^{+}>0>-\mu$ ) 


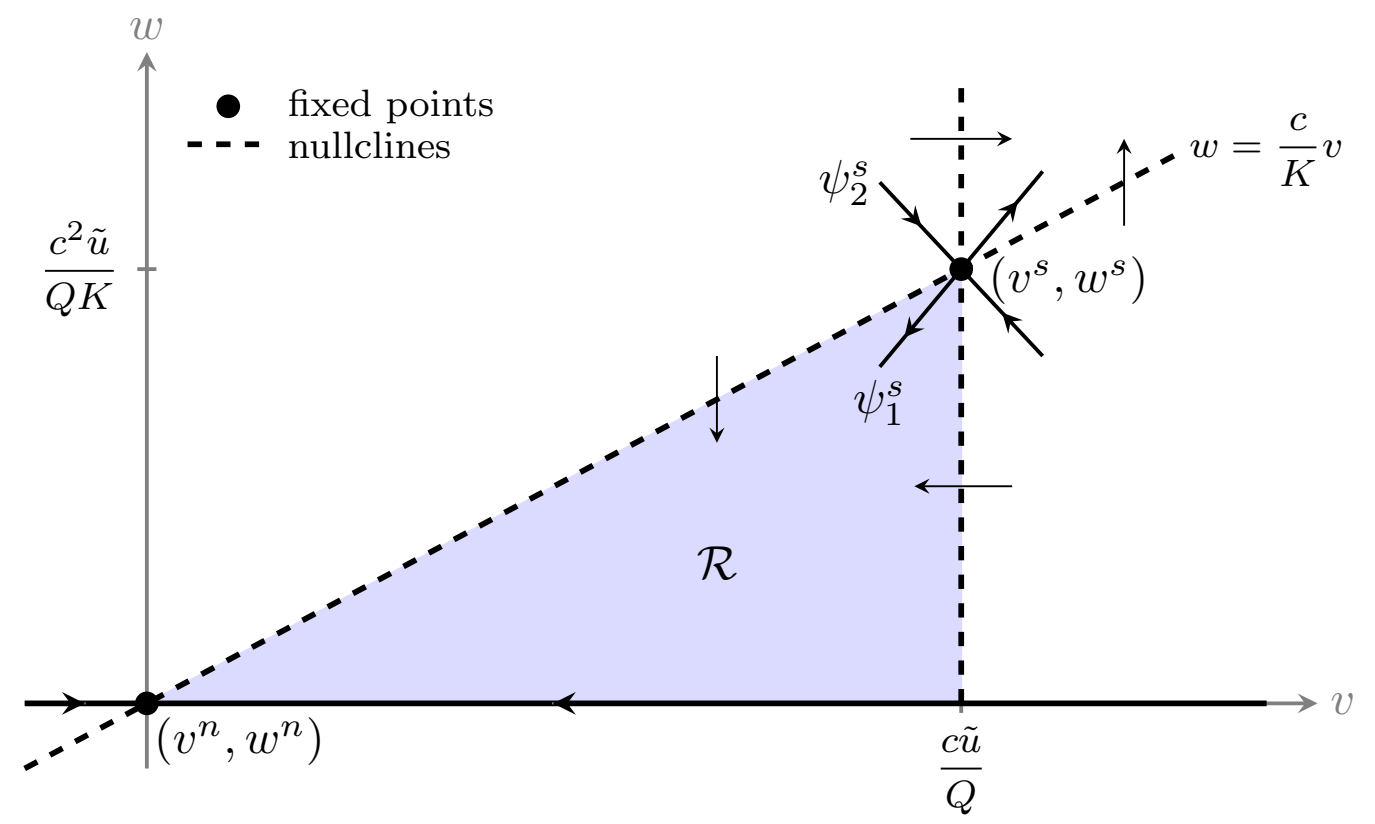

Figure 2: An illustration of the existence of a heteroclinic connection between $\left(v^{\mathrm{s}}, w^{\mathrm{s}}\right)$ and $\left(v^{\mathrm{n}}, w^{\mathrm{n}}\right)$ : the unstable trajectory leaving $\left(v^{\mathrm{s}}, w^{\mathrm{s}}\right)$ lies in $\mathcal{R}$ and since trajectories cannot leave $\mathcal{R}$ and there are no steady states inside $\mathcal{R}$ it must connect to the attracting steady state $\left(v^{\mathfrak{n}}, \mathcal{w}^{\mathfrak{n}}\right)$.

and there are no steady states inside $\mathcal{R}$, the Poincaré-Bendixson theorem [9] guarantees that the unstable trajectory leaving $\left(v^{s}, \mathcal{w}^{\mathrm{s}}\right)$ must connect with the stable node $\left(v^{\mathfrak{n}}, w^{\mathfrak{n}}\right)$; see Figure 2. The sign of $\mu-1$ determines whether this trajectory approaches $\left(v^{n}, w^{n}\right)$ via $\psi_{1}^{n}$ or $\psi_{2}^{n}$. 


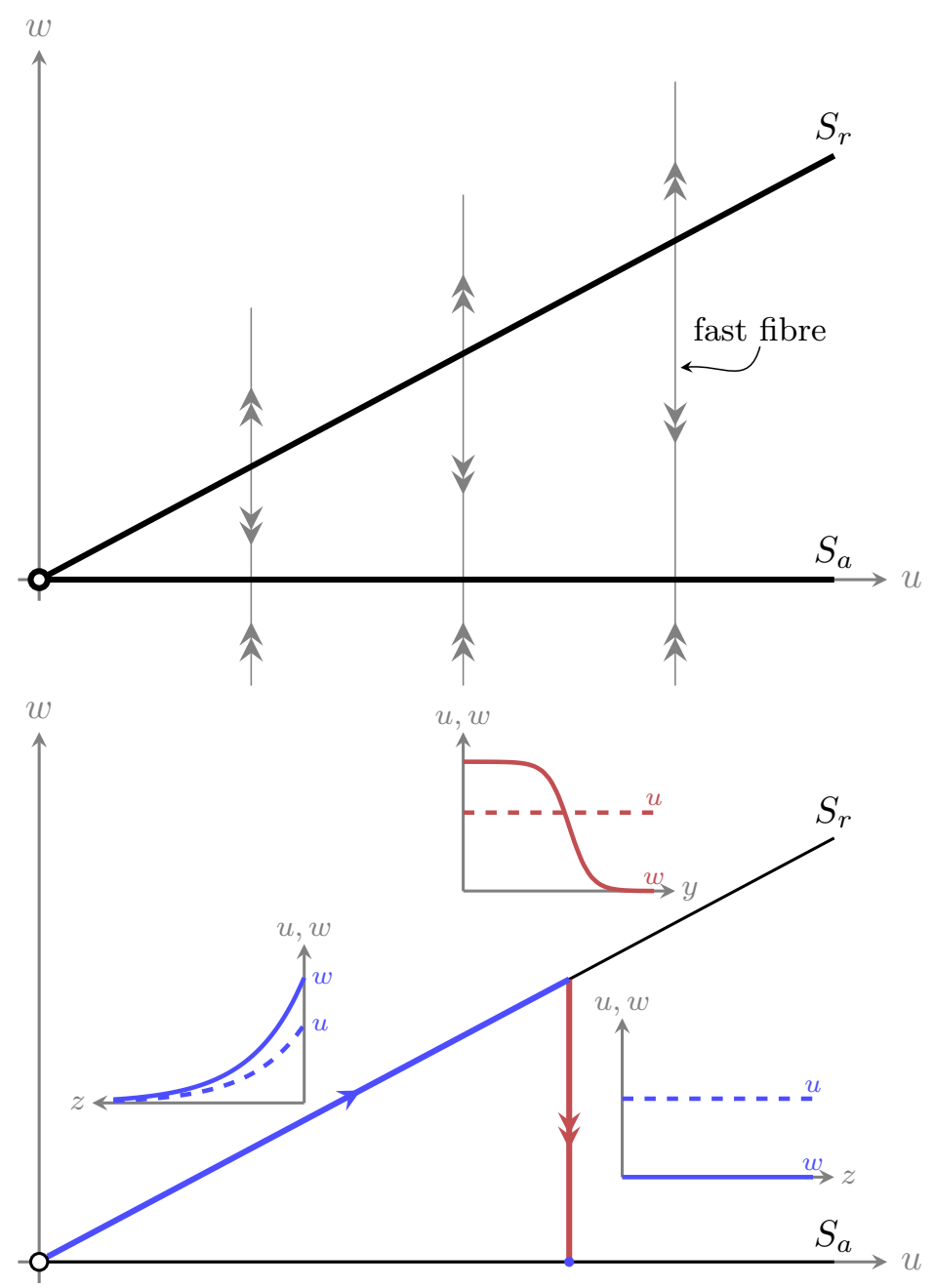

Figure 3: (top) The critical manifold $\mathbf{S}$, depicted in $(\mathfrak{u}, \mathfrak{w}$ )-space; and (bottom) the evolution of $u$ and $w$ in the different regions. The open circle at the origin signifies that the original system (1) has a singularity at this point. 


\subsection{Reduced problem}

The two algebraic constraints of (10) are equivalent to the steady states of (11) (with $\mathfrak{u}=\tilde{u}$ ). Consequently, the flow of the reduced problem is restricted to the one dimensional critical manifold

$$
\mathrm{S}=\left\{(\mathrm{u}, v, w) \mid v=\frac{\mathrm{K} w}{\mathrm{c}}, 0=w\left(\frac{\mathrm{Q} v}{\mathrm{u}}-\mathrm{c}\right)\right\},
$$

which consists of two branches

$$
S_{a}:=\{(u, v, w) \mid v=0, w=0\}
$$

and

$$
\mathrm{S}_{\mathrm{r}}:=\left\{(\mathrm{u}, v, w) \mid v=\frac{\mathrm{cu}}{\mathrm{Q}}, w=\frac{\mathrm{c}^{2} \mathrm{u}}{\mathrm{QK}}\right\} .
$$

The previous section demonstrated that $S_{a}$ and $S_{r}$ are normally hyperbolic: the steady states of the layer problem (11) have no eigenvalues with zero real part. Moreover, $S_{\mathrm{a}}$ restricted to $(v, w)$-space is attracting, while $S_{\mathrm{r}}$ restricted to $(v, w)$-space is repelling, hence the subscript choice. Finally, Lemma 3 shows that for fixed $u$ the layer flow as described by (11) provides connections between the points on $S_{r}$ and $S_{a}$ along fast fibres. An illustration is given in the top panel of Figure 3.

We consider the reduced flow on the two branches $S_{a}$ and $S_{r}$ separately. Firstly, on $S_{a}$ we have $u_{z}=0$. Therefore, there is no flow along $S_{a}$ and, using the asymptotic boundary conditions (3) with $\mathfrak{u}_{r}=1$, we have $(\mathfrak{u}, \boldsymbol{v}, \mathfrak{w})=(1,0,0)$. This also implies that $\mathfrak{u}=\tilde{u}=1$ along a fast fibre.

Secondly, on $S_{r}$ we have $u_{z}=c u / Q$, which is solved explicitly to give

$$
u(z)=e^{c\left(z+z_{*}\right) / Q},
$$

where $z_{*}$ is the constant of integration. Consequently,

$$
v(z)=\frac{\mathrm{c}}{\mathrm{Q}} e^{\mathrm{c}\left(z+z_{*}\right) / \mathrm{Q}}, \quad w(z)=\frac{\mathrm{c}^{2}}{\mathrm{QK}} e^{\mathfrak{c}\left(z+z_{*}\right) / \mathrm{Q}} .
$$


Thus, upon choosing $z_{*}=0$, in terms of the original variables $u$ and $\boldsymbol{w}$ and in the singular limit $\varepsilon \rightarrow 0$, the slow flow is

$$
u(z)=\left\{\begin{array}{ll}
e^{c z / Q} & \text { on } S_{r}, \\
1 & \text { on } S_{a},
\end{array} \quad \text { and } \quad w(z)= \begin{cases}\frac{c^{2}}{K Q} e^{c z / Q} & \text { on } S_{r} \\
0 & \text { on } S_{a}\end{cases}\right.
$$

\subsection{Singular heteroclinic orbits}

We now have enough information to construct heteroclinic orbits in the singular limit $\varepsilon \rightarrow 0$. These singular orbits are concatenations of components from the reduced and layer problems. We construct the waves in backward $z$.

In backward $z$, a solution begins on $S_{a}$ from the point $(u, v, w)=(1,0,0)$. Since there is no evolution of the slow variables on $S_{a}$, the only possibility is for the solution to switch onto a fast fibre of the layer problem. This connects the solution to the appropriate point on $S_{r}:(u, v, w)=\left(1, c / Q, c^{2} /(Q K)\right)$. Once back on $S_{\mathrm{r}}$, the slow flow of the reduced problem evolves the solution towards the initial state of the wave $(u, v, w)=(0,0,0)$; see the bottom panel of Figure 3 for an illustration.

The choice of $z_{*}$ in the previous section means that the jump between $S_{r}$ and $S_{a}$ occurs at $z=0$ and hence (12) is equivalent to (7).

\subsection{Heteroclinic orbits for $0<\varepsilon \ll 1$}

The persistence of the singular heteroclinic orbits for sufficiently small $0<$ $\varepsilon \ll 1$ to nearby smooth solutions of (8), and hence to travelling wave solutions of (1), is studied using Fenichel theory [4, 5]. In the following, we provide evidence that this theory can, in all likelihood, be applied to the problem at hand. 
Since $S_{r}$ and $S_{a}$ are normally hyperbolic, Fenichel's first theorem [6, e.g., and references therein] implies that these manifolds deform smoothly to $\mathcal{O}(\varepsilon)$-close, manifolds $S_{\mathrm{r}, \varepsilon}$ and $S_{\mathrm{a}, \varepsilon}$, which are locally invariant under the flow of $(8)^{1}$. In this case, the model is simple enough that we can compute these perturbed manifolds explicitly, to any order:

$$
\begin{aligned}
& \mathrm{S}_{\mathrm{r}, \varepsilon}=\left\{\left(\mathrm{u}_{\varepsilon}, v_{\varepsilon}, w_{\varepsilon}\right) \mid v_{\varepsilon}=\frac{c u_{\varepsilon}}{\mathrm{Q}-\varepsilon}, w_{\varepsilon}=\frac{\mathrm{c}^{2} \mathrm{u}_{\varepsilon}(\mathrm{Q}+\varepsilon(\mu-1))}{\mathrm{K}(\mathrm{Q}-\varepsilon)^{2}}\right\}, \\
& \mathrm{S}_{\mathrm{a}, \varepsilon}=\left\{\left(\mathrm{u}_{\varepsilon}, v_{\varepsilon}, w_{\varepsilon}\right) \mid v_{\varepsilon}=0, w_{\varepsilon}=0\right\}=\mathrm{S}_{\mathrm{a}} .
\end{aligned}
$$

It is not surprising that $S_{a, \varepsilon}=S_{a}$, since $S_{a}$ coincides with the background states of (1), which are not affected by $\varepsilon$. Consequently, the flow on $S_{a, \varepsilon}$ also remains unchanged, that is, there is no flow along $S_{a, \varepsilon}$. Furthermore, since $S_{r, \varepsilon} \rightarrow(0,0,0)$ as $u_{\varepsilon} \rightarrow 0$, the solution evolving on $S_{r, \varepsilon}$ will still connect (in backward $z$ ) to the initial state of the perturbed wave.

By Fenichel's second theorem ${ }^{2}$, the unstable manifold of $S_{r}, \mathcal{W}^{\mathrm{u}}\left(S_{r}\right)$, perturbs smoothly for $0<\varepsilon \ll 1$ to a nearby manifold $\mathcal{W}^{\mathrm{U}}\left(S_{r, \varepsilon}\right)$ that is locally invariant under the flow of (8). This also holds true for the stable manifold of $S_{a}$.

If $\mathcal{W}^{\mathrm{U}}\left(\mathrm{S}_{\mathrm{r}, \varepsilon}\right)$ and $\mathcal{W}^{\mathrm{S}}\left(\mathrm{S}_{\mathrm{a}, \varepsilon}\right)$ intersect, then Fenichel's third theorem ultimately guarantees the persistence of nearby fast fibres and, consequently, the persistence of the heteroclinic orbits. A sufficient condition for this intersection to occur is the transverse intersection of $\mathcal{W}^{\mathrm{U}}\left(\mathrm{S}_{\mathrm{r}}\right)$ and $\mathcal{W}^{\mathrm{S}}\left(\mathrm{S}_{\mathrm{a}}\right)$ since then the implicit function theorem implies persistence under small perturbations [6]. Doelman et al. [2] showed one possible technique for proving that $\mathcal{W}^{\mathrm{u}}\left(\mathrm{S}_{\mathrm{r}}\right)$ and $\mathcal{W}^{\mathrm{S}}\left(\mathrm{S}_{\mathrm{a}}\right)$ intersect transversely.

In conclusion, the solutions constructed in the singular limit appear to persist as nearby solutions of (1) for $D_{u}=\mu \varepsilon, D_{w}=\varepsilon$, with $\varepsilon$ sufficiently small,

${ }^{1}$ Fenichel theory only holds for compact manifolds. So, in principle, we first have to compactify the manifolds $S_{r}$ and $S_{a}$ by choosing an appropriate upper bound for $u$, for example, $u=2$.

${ }^{2}$ again after compactifying $S_{a, r}$. 
which connect to a nearby end state $(1+\mathcal{O}(\varepsilon), 0)$. However, since $(1)$ is invariant with respect to (6), we could rescale the perturbed wave to connect to the original end state of the unperturbed wave $(1,0)$ but with slightly different speed $\boldsymbol{c}(\varepsilon)$.

Remark 4. It is not clear a priori that GSPT extends to the singularity $(u, v, w)=(0,0,0)$. However, Doelman et al. [1] studied a generalised GiererMeinhardt equation, which also possesses a singularity within the domain of interest. As is the case here, this singularity lies on the boundary of the critical manifold and coincides with a fixed point of the reduced problem. They showed how to extend GSPT and Fenichel theory to points on the boundary of the critical manifold, assuming the system is well behaved as the solution approaches the singularity. Similar arguments to those presented by Doelman et al. [1] can, in principle, be used here to show that GSPT and Fenichel theory extend to the singularity.

Remark 5. The above results hold for $\mu=0$. Moreover, in this case we solve the layer problem explicitly:

$$
v=\frac{\mathrm{Kc} \tilde{u}}{\mathrm{QK}+\beta e^{c y}}, \quad w=\frac{\mathrm{c}^{2} \tilde{u}}{\mathrm{QK}+\beta e^{c y}},
$$

where $\beta$ is the integration constant.

\section{Conclusion}

Using GSPT, we provided arguments in support of the existence of travelling wave solutions to (1) with $\mathrm{D}_{\mathfrak{u}}=\mu \varepsilon, \mathrm{D}_{w}=\varepsilon$ and $\varepsilon$ sufficiently small. To leading order, these solutions are given by $\boldsymbol{u}_{\mathrm{r}}$ times the expressions for $\boldsymbol{u}$ and $\boldsymbol{w}$ stated in (12). Here, $\mathfrak{u}_{\mathrm{r}}$ is the asymptotic value of the $\boldsymbol{u}$ component of the wave for $z \rightarrow \infty$. This example acts to demonstrate the power of GSPT for studying the existence of travelling wave solutions of models such as the Keller-Segel model. 


\section{Acknowledgements}

This research was partially supported by the Australian Research Council's Discovery Project funding scheme (project number DP110102775).

\section{References}

[1] A. Doelman, R. A. Gardner, and T. J. Kaper. Large stable pulse solutions in reaction-diffusion equations. Indiana U. Math. J., 50(1):443-507, 2001. doi:10.1512/iumj.2001.50.1873 C412

[2] A. Doelman, P. van Heijster, and T. J. Kaper. Pulse dynamics in a three-component system: Existence analysis. J. Dyn. Differ. Equ., 21(1):73-115, 2009. doi:10.1007/s10884-008-9125-2 C411

[3] D. L. Feltham and M. A. J. Chaplain. Travelling waves in a model of species migration. Appl. Math. Lett., 13(7):67-73, 2000. doi:10.1016/S0893-9659(00)00079-3 C400, C401, C402, C403

[4] N. Fenichel. Persistence and smoothness of invariant manifolds for flows. Indiana U. Math. J., 21(3):193-226, 1972. doi:10.1512/iumj.1972.21.21017 C405, C410

[5] N. Fenichel. Geometric singular perturbation theory for ordinary differential equations. J. Differ. Equations, 31(1):53-98, 1979. doi:10.1016/0022-0396(79)90152-9 C405, C410

[6] G. Hek. Geometric singular perturbation theory in biological practice. J. Math. Biol., 60(3):347-386, 2010. doi:10.1007/s00285-009-0266-7 $\mathrm{C} 411$

[7] T. Hillen and K. J. Painter. A user's guide to PDE models for chemotaxis. J. Math. Biol., 58(1-2):183-217, 2009. doi:10.1007/s00285-008-0201-3 C400 
[8] C. K. R. T. Jones. Geometric singular perturbation theory. In Dynamical Systems, volume 1609, pages 44-118. Springer, 1995. doi:10.1007/BFb0095239 C401

[9] D. W. Jordan and P. Smith. Nonlinear Ordinary Differential Equations. Oxford University Press, 4th edition, 2007. ISBN: 9780199208258. http://ukcatalogue.oup.com/product/9780199208258.do C407

[10] T. J. Kaper. An introduction to geometric methods and dynamical systems theory for singular perturbation problems. In Proc. Sym. Appl. Math., volume 56, pages 85-131. American Mathematical Society, 1999. ISBN: 9780821809297.

http://www.ams.org/bookstore-getitem/item=PSAPM-56 C401

[11] E. F. Keller and L. A. Segel. Model for chemotaxis. J. Theor. Biol., 30(2):225-234, 1971. doi:10.1016/0022-5193(71)90050-6 C400

[12] E. F. Keller and L. A. Segel. Traveling bands of chemotactic bacteria: A theoretical analysis. J. Theor. Biol., 30(2):235-248, 1971. doi:0.1016/0022-5193(71)90051-8 C400

[13] M. J. Tindall, P. K. Maini, S. L. Porter, and J. P. Armitage. Overview of mathematical approaches used to model bacterial chemotaxis II: Bacterial populations. B. Math. Biol., 70(6):1570-1607, 2008. doi:10.1007/s11538-008-9322-5 C400

\section{Author addresses}

1. K. Harley, Mathematical Sciences School, Queensland University of Technology, Queensland 4001, Australia. mailto:ke.harley@student. qut. edu . au

2. P. van Heijster, Mathematical Sciences School, Queensland University of Technology, Queensland 4001, Australia. mailto:petrus.vanheijster@qut.edu.au 
3. G. J. Pettet, Mathematical Sciences School, Queensland University of Technology, Queensland 4001, Australia.

mailto:g.pettet@qut.edu.au 